\title{
Valores referenciales de posturografía basada en Nintendo Wii en población chilena
}

\section{Reference posturography values based on Nintendo Wii on Chilean population}

\author{
Juan Marambio G. ${ }^{1}$, Sebastián Castro M. ${ }^{1}$, Matías Gárate G. ${ }^{2}$, Ignacio Cortés F. ${ }^{2}$, \\ Javiera Corradini G. ${ }^{2}$, Leonardo Córdova P. ${ }^{2}$, Hayo Breinbauer K..$^{1,3,4}$
}

\section{Resumen}

Introducción: La posturografía es un método de exploración complementario para valorar el grado de desplazamiento del centro de gravedad (COP); no debe usarse de forma aislada al evaluar equilibrio, pero se acepta para seguimiento de la respuesta a tratamiento. Es de baja disponibilidad, por su alto costo comercial. Como alternativa se documenta la plataforma Wii Balance Board (WBB) de Nintendo para registro posturográfico. Objetivo: Describir valores de normalidad en parámetros posturográficos en población chilena sin patología vestibular, usando un registro simple y accesible. Material y Método: Estudio transversal de valores referenciales en herramienta para evaluación del equilibrio. Se registraron valores posturográficos con versión adaptada del Sensory Organization Test (SOT) a adultos sanos. Mediciones con software WBB Sway Program mediante WBB. Se midieron índices somatosensorial, visual y vestibular, y patrones de control postural en ejes anteroposterior y mediolateral durante SOT-adaptado. Resultados: Se obtuvieron valores de velocidad de desplazamiento del COP, área del COP y desviaciones estándar correspondientes para 4 situaciones del SOT-adaptado de 35 sujetos entre 18-65 años, 21 mujeres, 14 hombres. Discusión: WBB permitió evaluar estos parámetros en población sana, como aproximación a determinar rangos de referencia. Esta herramienta de uso rápido y accesible constituye una alternativa útil para determinar patrones de control postural en sujetos sanos. Presentamos valores a utilizar como rango referencial en nuestra población; se sugiere utilizar estos valores como objetivo terapéutico en rehabilitación vestibular en pacientes evaluados en conjunto con otros parámetros clínico-sintomáticos.

Palabras clave: Centro de gravedad, Wii balance board, test de organización sensorial, rango de normalidad.

\footnotetext{
Abstract

Introduction: Posturography is a complementary method for evaluating the center of pressure (COP) displacement; it should not be used in isolation when assessing balance, but it is accepted for follow-up of the treatment response. Due to its high commercial cost and low availability, we documented the Nintendo Wii Balance Board (WBB) as an alternative for posturography. Aim: To describe the normal range of posturography parameters in the Chilean population without vestibular pathology, using a simple and accessible registry method. Material and Method: Cross-sectional study of referential values in a balance assessment tool. Posturography values were registered using an adapted version of the Sensory Organization Test (SOT) on healthy adults. Measurements with WBB Sway Program software through WBB. With adapted-SOT, somatosensory, visual and vestibular indices, as well as anterior/posterior and mediolateral axes postural control patterns, were measured. Results: Values of displacement velocity of the COP, COP area and corresponding standard deviations were obtained for 4 SOT-adapted situations from 35 subjects between 18-65 years, 21 women, 14 men. Discussion: As an approach to determine referential range values, WBB allowed to evaluate these parameters in healthy population. To determine
}

'Servicio de
Otorrinolaringología, Hospital
San Juan de Dios. Santiago,
Chile.
Escuela de Medicina, Hospital
San Juan de Dios, Facultad
de Medicina, Universidad de
Chile. Santiago, Chile.
'Departamento de
Otorrinolaringología, Facultad
de Medicina, Universidad de
Chile. Santiago, Chile.
Departamento de
Neurociencia, Facultad de
Medicina, Universidad de
Chile. Santiago, Chile.
Los autores declaran no tener
conflictos de interés.
Recibido el 27 de abril de
2020 . Aceptado el 16 de
agosto de 2020.
Correspondencia:
Juan Marambio G.
Servicio de
Otorrinolaringología, Hospital
San Juan de Dios.
Huérfanos 3255. Santiago,
Chile.
Email: j_marambio@med.
uchile.com


healthy subjects postural control patterns this quick and accessible tool is a useful alternative. We present referential range values to use in our population; it is suggested to use these values as therapeutic objectives in vestibular rehabilitation in patients evaluated along with other clinical-symptomatic parameters.

Keywords: Center of pressure, Wii balance board, sensory organization test, normal range.

\section{Introducción}

La inestabilidad postural es un síntoma prevalente en la patología vestibular, neurológica, visual y ortopédica. Corresponde a una sensación de tambaleo o desvío de lado a lado al desplazarse, sin preponderancia direccional particular, producto de un déficit en el control del centro de gravedad, en relación a la base de apoyo de una persona ${ }^{1,2}$. Existen diversos métodos para evaluar la estabilidad postural, tanto, escalas cuantitativas como observacionales, así como test clínicos y/o funcionales con utilización de equipamiento ${ }^{3}$. Dentro de éstos últimos, la posturografía es un método promisorio que permite objetivar y cuantificar el grado de desplazamiento del centro de gravedad corporal ${ }^{4,5}$.

El uso de plataformas ha sido ampliamente utilizado para evaluar la estabilidad postural, basado en el registro de las fuerzas verticales en las esquinas de la plataforma, a partir de las cuales, se puede deducir la ubicación y movimientos de este centro de gravedad corporal y los efectos de las fuerzas utilizadas para mantener el equilibrio durante el registro $^{4,6,7}$. Sin embargo, esta herramienta no está exenta de limitaciones, y no se considera una técnica suficiente por sí sola para diagnostico o evaluación de afecciones otoneurológicas y musculoesqueléticas, evaluación de compensación o rehabilitación, o predicción de caídas ${ }^{8}$. Su uso es beneficioso cuando es apoyado por métodos complementarios, para darle vali$\mathrm{dez}^{5,8}$, especialmente si nuestro interés es el resultado terapéutico funcional ${ }^{9}$.

El equipamiento utilizado para realizar posturografía es de alto costo en versiones comerciales, alcanzando hasta $180.000 \mathrm{U} \$ \mathrm{D}^{10}$, lo que implica que esta herramienta sea de baja disponibilidad ${ }^{11,12}$. Por esta razón en los últimos años han surgido presentaciones alternativas más asequibles, permitiendo su mayor uso en clínica ${ }^{13,14}$. Se ha documentado el uso de la plataforma Wii Balance Board (WBB), accesorio de la consola Wii de Nintendo (Figura 1), para obtener datos posturográficos $\cos ^{5-7,13-15}$. Este trabajo realiza una aproximación experimental para determinar rangos de referencia posturográficos en nuestro medio usando el dispositivo de medición mencionado, a través de un programa de amplio acceso ${ }^{16}$, con el objetivo de ser utilizado en pacientes con patología otoneurológica en seguimiento y evaluación multidisciplinarios.

\section{Objetivo}

Describir valores de referencia en parámetros posturográficos en población chilena sin patología vestibular, usando un método simple y accesible de registro.

\section{Material y Método}

Estudio transversal de valores de normalidad en población sana de una herramienta de evaluación del equilibrio. Se registraron valores posturográficos a sujetos adultos mayores de 18 y menores de 65 años, sin patología vestibular, neurológica, visual y ortopédica. Las mediciones se realizaron entre los meses de agosto y noviembre de 2018. La participación de cada sujeto fue supeditada a un consentimiento informado previo a cada evaluación.

Para el registro de datos se aplicó una versión adaptada del Sensory Organization Test $(\mathrm{SOT})^{9,10}$, cuya versión original consiste en seis situaciones de evaluación de estabilidad postural combinando las siguientes variables en la realización de la prueba: superficie de la plataforma de apoyo firme versus apoyo inestable, clave visual presente versus ausente y clave visual normal versus alterada. Con la utilización de superficie inestable se altera el componente somatosensorial del equilibrio 
permitiendo evaluar de forma preferente los componentes visual y vestibular; al abolir la clave visual nos permite evaluar los componentes vestibular y somatosensorial aislados; y al alterar la clave visual también permite evaluar los componentes vestibular y somatosensorial aislados ${ }^{9}$. En el presente estudio utilizamos un SOT-adaptado, con cuatro situaciones que combinan algunas de estas variables (Tabla 1). La superficie inestable se realizó con una almohada sobre la plataforma y la clave visual se abolió solicitando al sujeto cerrar los ojos.

Las mediciones se realizaron con el software gratuito del grupo de investigación en alternativas de estudios funcionales de bajo costo del Dr. Ross Clark, WBB Sway Program ${ }^{16}$ (Figura 2) que registra valores posturográficos sobre la plataforma WBB (Figura 1) para reconocimiento del desplazamiento del centro de gravedad corporal (center of pressure o COP).

La información registrada correspondió a oscilaciones del COP durante las situaciones ajustadas del SOT, midiendo índices somatosensorial, visual y vestibular, y los patrones de control postural en los ejes anteroposterior (AP) y mediolateral (ML) de la plataforma. En una planilla Excel se registró para cada sujeto y cada situación del SOT-adaptado velocidad de desplazamiento del COP combinado para ejes AP y ML $(\mathrm{cm} / \mathrm{s})$, amplitud de desplazamiento AP $(\mathrm{cm})$ y amplitud de desplazamiento ML $(\mathrm{cm})$. A partir de los últimos dos valores se calculó el área del COP $\left(\mathrm{cm}^{2}\right)$. En base al área del COP para cada situación se calculó un valor promedio de las cuatro situaciones, considerando la situación 1 coeficiente uno, y las situaciones 2 a 4 coeficiente dos, obteniendo un área del COP global para la prueba.

\section{Resultados}

Se realizó posturografía aplicando el SOT-adaptado a 35 sujetos con rango de edad entre 18 a 65 años. Se evaluaron 21 mujeres y 14 hombres. Se obtuvieron medias de velocidad de desplazamiento del COP $(\mathrm{cm} / \mathrm{s})$, de área situacional del COP $\left(\mathrm{cm}^{2}\right)$ y de área global del COP $\left(\mathrm{cm}^{2}\right)$ para la muestra, con respectivas desviaciones estándar (Tablas 2 y 3). Los valores obtenidos de velocidad del desplazamiento del COP para las situaciones

\section{Tabla 1. Detalle de las situaciones para ejecutar el SOT*}

Situaciones del Sensory Organization Test-adaptado

1. Ojos abiertos, superficie firme

2. Ojos cerrados, superficie firme

3. Ojos abiertos, superficie inestable

4. Ojos cerrados, superficie inestable

*Adaptado del Sensory Organization Test ${ }^{9}$, originalmente elaborado para el dispositivo NeuroCom Balance Manager de Natus Medical Inc. ${ }^{2}$. Todas las tareas se realizan en posición erguida con pies juntos.

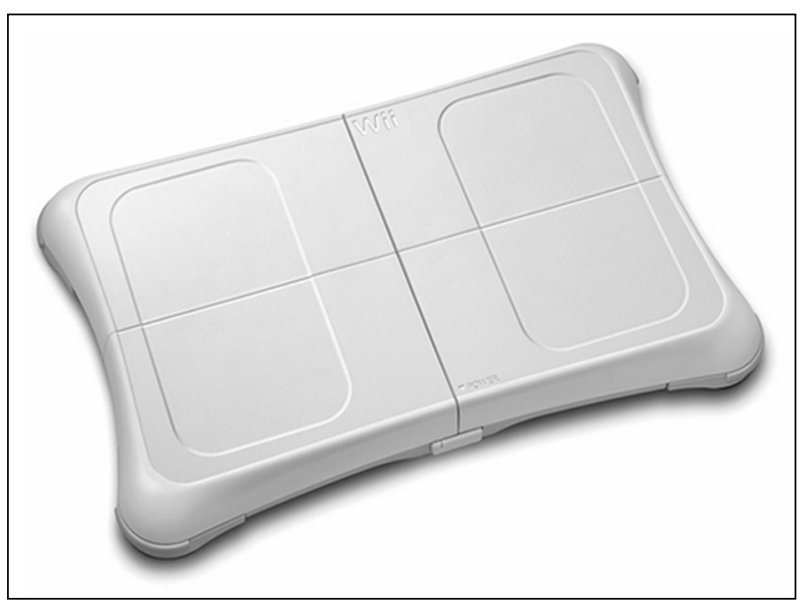

Figura 1. Wii Balance Board, accesorio de la consola Wii de Nintendo.

Tabla 2. Media de velocidad de desplazamiento del COP y área que abarca el COP, obtenidas en las situaciones del SOT*

\begin{tabular}{ccc}
\hline Situaciones SOT & Velocidad $(\mathbf{c m} / \mathbf{s})$ & Área COP $\left(\mathbf{c m}^{\mathbf{2}}\right)$ \\
Situación 1 & $1,34 \pm 0,44$ & $4,17 \pm 2,49$ \\
Situación 2 & $2,08 \pm 0,62$ & $8,15 \pm 3,75$ \\
Situación 3 & $1,93 \pm 0,52$ & $6,47 \pm 3,58$ \\
Situación 4 & $4,13 \pm 1,39$ & $23,32 \pm 12,93$ \\
\hline *Adaptado para la muestra examinada. &
\end{tabular}

Tabla 3. Área global promedio del COP para la muestra examinada obtenida a partir del área ponderada de cada situación del SOT*

$\begin{array}{cc}\text { S1-S4 ponderadas } & \text { Área global COP }\left(\mathbf{c m}^{2}\right) \\ (\mathrm{S} 1+2(\mathrm{~S} 2+\mathrm{S} 3+\mathrm{S} 4)) / 7 & 11,43 \pm 4,83\end{array}$

*Adaptado. 


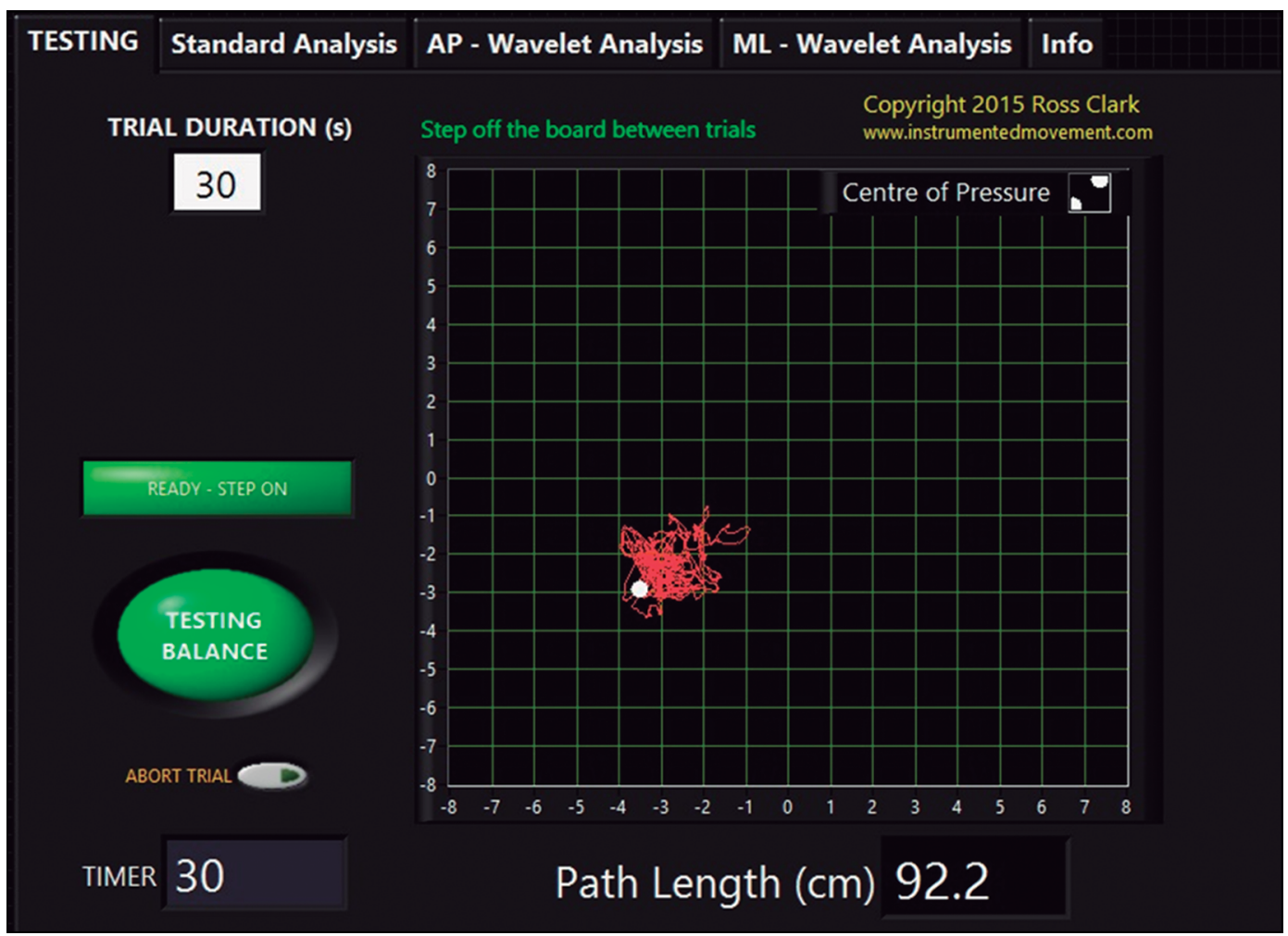

Figura 2. Interfaz gráfica del software WBB Sway Program para medir desplazamiento de COP registrado en Wii Balance Board, disponible de forma gratuita en www.rehabtools.org. Presenta un recuadro cuadriculado que representa la plataforma en sus ejes anteroposterior (AP) y mediolateral (ML). El COP es representado por un punto blanco, mientras que el desplazamiento de éste se registra visualmente con un trazado rojo. En pestañas correspondientes (Standard Analysis, AP-Wavelet Analysis, ML-Wavelet Analysis) se obtiene el cálculo numérico de velocidad de desplazamiento y área del COP.

del SOT-adaptado en cada uno de los sujetos se registraron en $\mathrm{cm} / \mathrm{s}$. Por otro lado, los valores del área del COP se registraron en $\mathrm{cm}^{2}$. Se obtuvo la media de la muestra con desviación estándar para estas dos variables.

\section{Discusión}

El uso de la plataforma WBB permitió evaluar de forma rápida y accesible parámetros posturográficos en población sin patología vestibular, neurológica, visual u ortopédica, como una aproximación a determinar rangos de referencia. Se pueden determinar patrones de control postural estático con esta herramienta, constituyendo una alternativa válida y de características de confiabilidad similares a las plataformas de posturografía comerciales tí- picas $^{7}$, lo que la establece como un instrumento de utilidad comprobada y adecuada para el entorno clínico, a una fracción del costo de los métodos tradicionales ${ }^{17}$, permitiendo valorar el equilibrio estático de una forma más global, como resultado de la integración de todos los componentes del equilibrio. Desde esta potencialidad, WBB permite guiar procesos de rehabilitación vestibular y del equilibrio con objetividad de una forma accesible.

Este instrumento también ha sido probado en la evaluación de distribución de fuerzas de apoyo en patología musculoesquelética, mostrándose también como una alternativa válida y de confiabilidad similares a las plataformas originales $^{3,18}$. Esta aplicación ha sido utilizada en diversas áreas, otorgando información a bajo costo que permite mejorar y ampliar la difusión de la evaluación del equilibrio ${ }^{15}$ y de 
la distribución de fuerzas ${ }^{18}$, lo que favorece, no solo una mejora en los procesos terapéuticos de cada paciente, sino la posibilidad de hacer investigación objetiva, de bajo costo y masiva en relación a terapia de equilibrio. Sin embargo, debe utilizarse con cuidado al evaluar distribución de peso de menor carga, como en la evaluación de la distribución de fuerza en extremidades superiores, dado que la validez es pobre comparada a una plataforma tradicional en estos casos en particular ${ }^{19}$. También el enfoque debe darse mayormente en el valor de área del COP por sobre la velocidad de desplazamiento de éste, dado que se describe una mayor precisión de la WBB en la trayectoria (que determina el área del COP) por sobre la velocidad medida ${ }^{20}$. Por otro lado, se debería usar un mismo equipo para obtener resultados más confiables, ya que está descrito que la incertidumbre de localización del COP entre distintos equipos de $\mathrm{WBB}$ es de $\pm 4,1$ $\mathrm{mm}$ versus $\pm 1,5 \mathrm{~mm}$ en una misma plataforma $\mathrm{WBB}^{20-24}$, así como la fiabilidad entreplataformas es de $47 \%$ vs $88 \%$ en test-retest en misma WBB en sujetos sanos ${ }^{25}$.

Por último, se debe tener en cuenta que, si bien las características que hemos descrito favorecen su uso clínico en varios ámbitos, no reemplaza en la función diagnóstica a los métodos de evaluación tradicional del equilibrio, ya que carece de la misma exactitud, precisión y confiabilidad de los dispositivos médicos ${ }^{21}$. Pero como virtud principal, permite guiar con objetividad los procesos de rehabilitación vestibular y del equilibrio de casos en los que ya se ha realizado el proceso diagnóstico, utilizándola de forma complementaria a otros métodos de evaluación ${ }^{8,22,23}$.

Sin embargo, reconocemos que nuestro tamaño muestral es bajo, y que el rango etáreo de la muestra no abarca la totalidad de poblaciones de interés. A pesar de esto, creemos que el valor de este artículo es dar pie e incentivar el uso de una herramienta tan poderosa como de fácil acceso como lo es un posturógrafo basado en Wii. Nuestra sugerencia es utilizar estos valores de rangos referenciales del SOT-adaptado medido en la plataforma WBB como objetivo o meta terapéutica en procesos de rehabilitación vestibular, en pacientes evaluados en conjunto con parámetros clínico-sintomáticos que objetiven un proceso de mejora clínica, como escalas de limitación funcional autopercibida (dizziness hándicap inventory) y pruebas de rendimiento físico (Berg balance scale, test de agudeza visual dinámica, timed up and $g o)^{3,9,23}$, realizando las mediciones de forma longitudinal en el tiempo en una misma plataforma $\mathrm{WBB}^{24,25}$.

\section{Conclusión}

El uso del WBB aplicando el software WBB Sway Program constituye un método asequible y fácilmente reproducible para realizar posturografía. Esta herramienta resulta un elemento de apoyo para monitorizar la evolución en la rehabilitación de patología del equilibrio, que creemos es de utilidad. Los valores de velocidad y área del COP en el SOT-adaptado presentados en este trabajo constituyen un aporte inicial a la terapia del equilibrio, pudiendo utilizarse como valores de objetivo terapéutico en nuestra población en tratamiento de rehabilitación vestibular.

\section{Bibliografía}

1. Staab J, Eckhardt-Henn A, Horii A, et al. Diagnostic criteria for persistent postural-perceptual dizziness (PPPD): Consensus document of the committee for the classification of vestibular disorders of the Bárány Society. J Vestib Res. 2017;27:191-208.

2. Pletcher ER, Williams VJ, Abt JP, et al. Normative data for the NeuroCom Sensory Organization Test in US military special operations forces. J Athl Train. 2017;52(2):129-136. doi: 10.4085/1062-6050-52.1.05.

3. Piirtola M, Era P. Force Platform measurements as predictors of falls among older people - A review. Gerontology. 2006;52:1-16. doi: 10.1159/000089820.

4. Chaudhry H, Bukiet B, Ji Z, Findley T. Measurement of balance in computer posturography: Comparison of methods-A brief review. J Bodyw Mov Ther. 2011;15(1):82-91. doi: 10.1016/j.jbmt.2008.03.003.

5. Falls C. Videonystagmography and posturography. Adv Otorhinolaryngol. 2019; 82:32-8. doi: 10.1159/000490269.

6. Petró B, Papachatzopoulou A, Kiss RM. Devices and tasks involved in the objective assessment of standing dynamic balancing - A systematic literature review. PLoS One. 2017;12(9):e0185188. doi: 10.1371/journal. pone.0185188.

7. Clark RA, Mentiplay BF, Pua YH, Bower KJ. Reliability and validity of the Wii Balance Board 
for assessment of standing balance: A systematic review. Gait Posture. 2018;61:40-54. doi: 10.1016/j. gaitpost.2017.12.022.

8. Kingma H, Gauchard GC, de Waele C, et al. Stocktaking on the development of posturography for clinical use. J Vestib Res. 2011;21(3):117-125. doi: 10.3233/VES-2011-0397.

9. Clendaniel RA. Outcome measures for assessment of treatment of the dizzy and balance disorder patient. Otolaryngol Clin North Am. 2000;33(3):519-533. doi: 10.1016/s0030-6665(05)70225-5.

10. Shirley Ryan AbilityLab. Rehabilitation measures database, Sensory organization test 2013. Disponible en: https://www.sralab.org/rehabilitation-measures/ sensory-organization-test. Consultado el: 23 de abril de 2020.

11. Visser JE, Carpenter MG, van der Kooij H, Bloem BR. The clinical utility of posturography. Clin Neurophysiol. 2008;119(11):2424-2436. doi: 10.1016/j. clinph.2008.07.220.

12. Freeman L, Gera G, Horak FB, Blackinton MT, Besch M, King L. Instrumented test of sensory integration for balance: A validation study. $J$ Geriatr Phys Ther. 2018;41(2):77-84. doi: 10.1519/ JPT.0000000000000110.

13. Dutta A, Chugh S, Banerjee A, Dutta A. Point-ofcare-testing of standing posture with Wii balance board and microsoft kinect during transcranial direct current stimulation: A feasibility study. NeuroRehabilitation. 2014;34:789-98. doi: 10.3233/ NRE-141077.

14. Littrell ME, Chang YH, Selgrade BP. Development and assessment of a low-cost clinical gait analysis system. J Appl Biomech. 2018;10:1-19. doi: 10.1123/ jab.2017-0370.

15. Mengarelli A, Cardarelli S, Strazza A, Di Nardo F, Fioretti S, Verdini F. Validity of the Nintendo Wii Balance Board for the assessment of balance measures in the functional reach test. IEEE Trans Neural Syst Rehabil Eng. 2018;26(7):1400-6. doi: 10.1109/ TNSRE.2018.2843884.

16. Clark RA, Pua YH. Physical function assessment tools, The Nintendo Wii Balance Board 2015. Disponible en: http://www.rehabtools.org/wiibalance-board.html. Consultado el: 18-11-2018.

17. Clark RA, Bryant AL, Pua Y, McCrory P, Bennell K,
Hunt M. Validity and reliability of the Nintendo Wii Balance Board for assessment of standing balance. Gait Posture. 2010;31:307-310. doi: 10.1016/j. gaitpost.2009.11.012.

18. Abujaber S, Gillispie G, Marmon A, Zeni J. Validity of the Nintendo Wii Balance Board to assess weight bearing asymmetry during sit-to-stand and return-tosit task. Gait Posture. 2015;41:676-682. doi: 10.1016/j. gaitpost.2015.01.023.

19. Eshoj H, Juul-Kristensen B, Jørgensen RG, Søgaard K. Reproducibility and validity of the Nintendo Wii Balance Board for measuring shoulder sensorimotor control in prone lying. Gait Posture. 2017;52:211-216. doi: 10.1016/j.gaitpost.2016.12.003.

20. Huurnink A, Fransz DP, Kingma I, Van Dieën JH. Comparison of a laboratory grade force platform with a Nintendo Wii Balance Board on measurement of postural control in single-leg stance balance tasks. J Biomech. 2013; 46:1392-1395. doi: 10.1016/j. jbiomech.2013.02.018.

21. Leach JM, Mancini M, Peterka RJ, Hayes TL, Horak FB. Validating and calibrating the Nintendo Wii Balance Board to derive reliable center of pressure measures. Sensors. 2014;14:18244-18267. doi: 10.3390/s141018244.

22. Sample RB, Kinney AL, Jackson K, Diestelkamp W, Bigelow KE. Identification of key outcome measures when using the instrumented timed up and go and/or posturography for fall screening. Gait Posture. 2017; 57:168-171. doi: 10.1016/j.gaitpost.2017.06.007.

23. Cabral KN, Brech GC, Alonso AC, et al. Posturographic measures did not improve the predictive power to identify recurrent falls in community-dwelling elderly fallers. Clinics (Sao Paulo). 2020;75:e1409. doi: 10.6061/clinics/2020/ e1409.

24. Bartlett HL, Ting LH, Bingham JT. Accuracy of force and center of pressure measures of the Wii Balance Board. Gait Posture. 2014; 39:224-228. doi: 10.1016/j. gaitpost.2013.07.010.

25. Castelli L, Stocchi L, Patrignani M, Sellitto G, Giuliani M, Prosperini L. We-Measure: Toward a low-cost portable posturography for patients with multiple sclerosis using the commercial Wii balance board. J Neurol Sci. 2015; 359:440-444. doi: 10.1016/j. jns.2015.10.016. 